\title{
SUBGROUPS OF FREE PRODUCTS WITH AMALGAMATED SUBGROUPS: A TOPOLOGICAL APPROACH
}

\author{
BY
}

\section{J. C. CHIPMAN $\left({ }^{1}\right)$}

ABSTRACT. The structure of an arbitrary subgroup of the limit of a group system is shown to be itself the limit of a group system, the elements of which can be described in terms of subgroups of the original group system.

Introduction. It is the intent of this paper to describe the structure of an arbitrary subgroup of the limit of a group system in terms of subgroups of the members of that system. The description that is obtained is given by the following theorem:

Theorem. Let $H$ be any subgroup of the limit of the group system ( $G_{\alpha}$, $\left.H_{\alpha \beta}, i_{\alpha \beta}: \alpha, \beta \in \Lambda\right)$. Then $H$ is itself the limit of a group system. Each of the factors of $H$ is the free product of a conjugate of a subgroup of one of the $G_{\alpha}$ and a free group (which may be trivial). Each of the amalgamating groups is the free product of a conjugate of a subgroup of one of the $H_{\alpha \beta}$ and a free group (which may be trivial).

The general approach to this problem will be topological. That is to say, a complex $K$ whose fundamental group is the limit of the given group system and whose topological composition parallels the algebraic composition of that group system will be constructed. The structure of a subgroup $H$ may then be determined by computations of the fundamental group of a covering space of $K$.

\$ will set the notation and terminology that will be used throughout, and $\S$ II will give the construction of the model complex $K$ with some properties of its covering spaces. $\$$ III will give the proof of the main theorem, and will discuss the relation of the approach taken here to results previously obtained in similar settings.

Received by the editors September 23, 1971 and, in revised form, November 22, 1972.

AMS (MOS) subject classifications (1970). Primary 20E30, 20F15; Secondary 55A05, 55A10.

Key words and phrases. Group system, fundamental group, covering space, tree product, HNN group, generalized free product.

(1) Part of this material was included in the author's Ph.D. dissertation submitted to Dartmouth College and supervised by Professor Edward M. Brown. 
I. The algebraic construction being studied here is the limit of a group system $\left(G_{\alpha}, H_{\alpha \beta}, i_{\alpha \beta}: \alpha, \beta \in \Lambda\right)$. By this we mean that there is an indexed family of groups $\left\{G_{\alpha}: a \in \Lambda\right.$; ; associated with every pair of groups $G_{\alpha}, G_{\beta}$ there is a group $H_{\alpha \beta}$ and homomorphisms $i_{\alpha \beta}: H_{\alpha \beta} \rightarrow G_{\alpha}$ and $i_{\beta \alpha}: H_{\alpha \beta} \rightarrow G_{\beta}$. The groups $G_{\alpha}$ will be referred to as the factors while the groups $H_{\alpha \beta}$ will be referred to as the amalgamating groups. By the limit of the group system we mean the limit of the diagram of the group system in the categorical sense. That is

Definition I.1. ( $\left.G, g_{\alpha}: G_{a} \rightarrow G, b_{\alpha \beta}: H_{\alpha \beta} \rightarrow G ; \alpha, \beta \in \Lambda\right)$ where $g_{\alpha} i_{\alpha \beta}=$ $b_{\alpha \beta}=g_{\beta} i_{\beta a}$ is the limit of the group system $\left(G_{\alpha}, H_{\alpha \beta}, i_{\alpha \beta}: \alpha, \beta \in \Lambda\right)$ provided that, for any group $M$ and family of homomorphisms $k_{\alpha}: G_{\alpha} \rightarrow M, l_{\alpha \beta}: H_{\alpha \beta} \rightarrow M$ such that $l_{\alpha \beta}=k_{\alpha} i_{\alpha \beta}=k_{\beta} i_{\beta a}$, there exists a unique homomorphism $k: G \rightarrow M$ such that $k_{\alpha}=k g_{a}$ and $l_{\alpha \beta}=k b_{\alpha \beta}$ for all $\alpha, \beta \in \Lambda$.

In other settings such structures have been referred to as generalized free products with amalgamation, depending on the conditions that have been imposed on the groups $H_{\alpha \beta}$, the $i_{\alpha \beta}$, or the $k_{\alpha}$, for example [5]. One particular case that will be of interest later on is where the $b_{\alpha \beta}$ are monomorphisms and the diagram is a tree; this is what is called a tree product [3].

Another type of group structure will be encountered later in $\$$ III. Let $K$ be a group and $\left\{\phi_{i}\right\}$ a collection of monomorphisms of subgroups $L_{i}$ of $K$ into $K$.

Definition I.2. The group $G$ is called an HNN group with base $K$ and associated subgroups $L_{i}, \phi_{i}\left(L_{i}\right)$ if $G$ has a group presentation of the form

$$
G=\left(t_{1}, t_{2}, \ldots, \operatorname{gen}(K) ; \operatorname{rel}(K), t_{1} L_{1} t_{1}^{-1}=\phi_{1}\left(L_{1}\right), t_{2} L_{2} t_{2}^{-1}=\phi_{2}\left(L_{2}\right), \ldots\right) \text {. }
$$

For the computation of the fundamental group of the covering space we will need to make reference to types of coverings that a space may have; this is done via the following:

Definition I.3. Let $\left\{K_{a}: \alpha \in \Lambda\right\}$ be a covering of the complex $K$ by connected subcomplexes of $K$. The completed cover $\Sigma$ associated with $\left\{K_{a}: a \in \Lambda\right\}$ is the covering whose members consist of $A$ where $A=K_{\alpha}$ for some $\alpha$ or $A$ is an arc component of $B_{1} \cap B_{2}$ where $B_{1}, B_{2} \in \Sigma$.

Of particular interest will be those completed covers which satisfy the additional conditions given by

Definition I.4. An $n$-stage cover $\Sigma$ of the complex $K$ consists of a finite nonempty sequence $\lambda_{0}, \ldots, \lambda_{n}$ of collections of subcomplexes of $K$ such that

(i) $\lambda_{0}$ is a cover of $K$ by connected subcomplexes of $K$.

(ii) $\lambda_{i}$ is the set of all path components of pairwise intersections $A_{1} \cap A_{2}$, where $A_{1}, A_{2} \in \lambda_{i-1}$.

(iii) If $A \in \lambda_{i}$, then $A-U \lambda_{i+1}$ is connected and nonempty.

(iv) The set of path components of $\bigcup \lambda_{n}$ is equal to $\lambda_{n}$ (or equivalently, $\lambda_{n}$ consists of disjoint sets). 
For convenience, we write $\Sigma=\bigcup_{i=0}^{n} \lambda_{i}$.

II. In this section the necessary topological constructions will be considered. Of primary importance will be the topological model for the algebraic problem under discussion. Its formulation is given as follows:

Theorem II.1. Let $G$ be the limit of the group system $\left(G_{\alpha}, H_{\alpha \beta}, i_{\alpha \beta}: \alpha, \beta\right.$ $\epsilon \Lambda)$; then there is a complex $K$ with $\pi_{1}(K) \approx G$. Moreover, $K$ has a covering by subcomplexes $\left\{K_{\alpha}: \alpha \in \Lambda\right\}$ such that $\pi_{1}\left(K_{\alpha}\right) \approx G_{\alpha}, \pi_{1}\left(K_{\alpha} \cap K_{\beta}\right) \approx H_{\alpha \beta}$, and such that the homomorphisms induced by the inlcusion maps $K_{\alpha} \cap K_{\beta} \rightarrow K_{\alpha}$ and $K_{\alpha} \cap K_{\beta} \rightarrow K_{\beta}$ are $i_{\alpha \beta}$ and $i_{\beta \alpha}$ respectively.

Proof. Let $\left\{L_{\alpha}, L_{\alpha \beta}: \alpha, \beta \in \Lambda\right\}$ be a family of complexes with $\pi_{1}\left(L_{\alpha}, v_{\alpha}\right)$ $\approx G_{\alpha}, \pi_{1}\left(L_{\alpha \beta}, v_{\alpha \beta}\right) \approx H_{\alpha \beta}$. We remark at this point that each of these complexes will be considered to be a wedge of 1 -spheres with appropriate discs attached. Select points $v_{0}, v_{1}$ not in the spaces so formed and introduce arcs from $v_{1}$ to each of the $v_{a}$ and from $v_{0}$ to each of the $v_{\alpha \beta}$. Introduce an arc $s$ from $v_{0}$ to $v_{1}$, and call the resulting complex $K^{\prime}$. Note that there are unique paths $s_{\alpha \beta}$ and $s_{\beta \alpha}$ from each $v_{\alpha \beta}$ to $v_{\alpha}$ and $v_{\beta}$. A family of discs $D$ is now attached to $K^{\prime}$ as follows. For each $\alpha, \beta \in \Lambda$ and each $a \in \mathrm{a}$, a set of generators for $H_{\alpha \beta}$, let $\gamma_{0}, \gamma_{1}$ and $\gamma_{2}$ be representative loops for $a, i_{\alpha \beta}(a)$, and $i_{\beta \alpha}(a)$ in $L_{\alpha \beta}$, $L_{\alpha}$, and $L_{\beta}$ respectively. Discs are attached by identifying boundaries with the paths $\gamma_{0}^{-1} s_{\alpha \beta} \gamma_{1} s_{\alpha \beta}^{-1}$ and $\gamma_{0}^{-1} s_{\beta \alpha} \gamma_{2} s_{\beta a}^{-1}$. This is done for each $\alpha, \beta$ and a. Let $K$ be the complex obtained.

Now for each $\alpha \in \Lambda$, let $K_{\alpha}$ be the complex consisting of the union of $L_{\alpha^{\prime}}$ $L_{\alpha \beta}$ for all $\beta$ and those discs which have been introduced for the $i_{\alpha \beta}$. We claim that $\pi_{1}\left(K_{\alpha}\right) \approx G_{\alpha}$. To see this we consider $K_{\alpha}$ to be constructed from $L_{\alpha}$ by attaching the appropriate discs from $\mathscr{D}$ followed by the addition of the $L_{\alpha \beta}$. Since $L_{\alpha}$ is clearly a deformation retract of $L_{\alpha} \cup\left(\mathfrak{I} \cap K_{\alpha}\right)$, the result holds for the first stage of the construction. Now because of the way that the $L_{\alpha \beta}$ are constructed, the addition of a $L_{\alpha \beta}$ consists of attaching discs to $L_{\alpha} \cup\left(\mathfrak{P} \cap K_{\alpha}\right)$. Therefore it suffices to show that the addition of such a disc does not increase the class of null homotopic loops in $L_{\alpha} \cup\left(\vartheta \cap K_{\alpha}\right)$. Let $D$ be such a disc giving rise to the relation $r_{\alpha \beta}$ in $H_{\alpha \beta}$ and let $\gamma$ be a representative loop for $r_{\alpha \beta}$. Because of the way that the discs $\mathcal{I}$ have been attached we have that $\gamma$ is homotopic to $\gamma^{\prime}$, a representative loop for $i_{\alpha \beta}\left(r_{\alpha \beta}\right)$ in $L_{\alpha}$. Since $i_{\alpha \beta}$ is a homomorphism, $i_{\alpha \beta}\left(r_{\alpha \beta}\right)=1$ and so $\gamma^{\prime}$ was already null homotopic in $L_{\alpha}$ and hence in $L_{\alpha} \cup\left(\vartheta \cap K_{\alpha}\right)$. Therefore $\pi_{1}\left(K_{\alpha}\right) \approx \pi_{1}\left(L_{\alpha}\right) \approx G_{\alpha}$.

For $a$ different from $\beta, K_{\alpha} \cap K_{\beta}$ consists of $L_{\alpha \beta}$, the arc from $v_{\alpha \beta}$ to $v_{0}$, and the arc $s$. Thence $\pi_{1}\left(K_{\alpha} \cap K_{\beta}\right) \approx \pi_{1}\left(L_{\alpha \beta}\right) \approx H_{\alpha \beta}$. Inherent in the construction is the fact that the inclusion maps will induce the desired homomorphisms $i_{\alpha \beta}$ and $i_{\beta \alpha}$ for each $\alpha, \beta \in \Lambda$. 
To show that $\pi_{1}(K) \approx G$ a maximal tree is chosen for $K$. The maximal tree is constructed by first taking the arc $s$, the arcs from $v_{\alpha \beta}$ to $v_{0}$, and maximal trees in each of the $L_{\alpha \beta}$. In each $K_{\alpha}$ this tree is then extended to a maximal tree $T_{\alpha}$. Note that in each $T_{\alpha}$ the path from a vertex of any of the $L_{\alpha \beta}$ to a vertex in $L_{\alpha}$ or the interior of any of the attached discs of $\mathcal{P}$ must contain the arc s. Thus $\bigcup_{\alpha} T_{a}$ is a tree in $K$ and is in fact a maximal tree in $K$. This maximal tree meets every member of the completed cover generated by the $K_{a}$ in a maximal tree which suffices by [1] to give $\pi_{1}(K)$ as the limit of the system of fundamental groups of the members of the cover. Since the fundamental group of the arc $s$ is trivial we then have $\pi_{1}(K) \approx G$ as was desired.

Corollary II.2. Let $\Sigma$ be the associated completed cover of $\left\{K_{a}: a \in \Lambda\right\}$ constructed above and define

(i) $\lambda_{0}=\left\{K_{\alpha}: \alpha \in \Lambda\right\}$,

(ii) $\lambda_{1}=\left\{L_{\alpha \beta} \cup s \cup\right.$ arc from $v_{\alpha \beta}$ to $\left.v_{0}: \alpha, \beta \in \Lambda\right\}$,

(iii) $\lambda_{2}=\{s\}$.

Then $\Sigma$ is a 2-stage cover of $K$.

Proof. This is immediate from the construction of $K$.

For our purposes we will be interested in studying an arbitrary covering space $(\tilde{K}, p)$ of $K$ and finding a covering of $\widetilde{K}$ that will facilitate the computation of its fundamental group. An obvious choice is obtained by considering the arc components of $p^{-1}\left(K_{\alpha}\right)$ for $\alpha \in \Lambda$. It is not difficult to check that the completion of this cover, $\widetilde{\Sigma}$, will consist of the arc components of $p^{-1}\left(L_{\alpha \beta} \cup s \cup\right.$ arc from $v_{\alpha \beta}$ to $\left.v_{0}\right)$ and the arc components of $p^{-1}(s)$. This cover will also be a 2 -stage cover. Take $\tilde{\lambda}_{0}$ to be the arc components of $p^{-1}\left(K_{\alpha}\right), \tilde{\lambda}_{1}$ to be the arc components of $p^{-1}\left(L_{\alpha \beta} \cup s \cup\right.$ arc from $v_{\alpha \beta}$ to $\left.v_{0}\right)$, and $\tilde{\lambda}_{2}$ to be the arc components of $p^{-1}(s)$. The only detail that is not immediate is that, for $A \in \widetilde{\lambda}_{0}, A-\bigcup \widetilde{\lambda}_{1}$ will be connected. To see this choose points $\tilde{x}, \tilde{y} \in A$, project down to points $p(\tilde{x}), p(\tilde{y})$ in $K_{\alpha}$ for some $\alpha$, and use the fact that $\Sigma$ is a 2 -stage cover to find a path in $K_{\alpha}-\bigcup \lambda_{1}$ from $p(\tilde{x})$ to $p(\tilde{y})$. This path can then be lifted to a path in $A$ from $\tilde{x}$ to $\tilde{y}$. This gives us the final topological fact needed to study the algebraic problem, namely:

Corollary 1.3. Let $(\widetilde{K}, p)$ be an arbitrary covering space of $K$. Then $\widetilde{K}$ bas a 2-stage covering consisting of the arc components of $p^{-1}\left(K_{\alpha}\right)$, $p^{-1}\left(L_{\alpha \beta} \cup s \cup\right.$ arc from $v_{\alpha \beta}$ to $\left.v_{0}\right)$, and $p^{-1}(s)$ as $\tilde{\lambda}_{0}, \tilde{\lambda}_{1}$, and $\tilde{\lambda}_{2}$ respectively.

III. In this section the structure of various subgroups of the limit of a group system will be studied. An attempt will also be made to consider various algebraic conditions and results that have been given before and to interpret the ir 
their topological counterparts. As with most topological treatments of algebraic problems the key tool is that of covering spaces and the basic theorem which assures that for any subgroup $H$ of $\pi_{1}\left(K, v_{0}\right)$ there exist a covering space $(\widetilde{K}, p)$ of $K$ and basepoint $\widetilde{v}_{0} \in \widetilde{K}$ such that $\pi_{1}\left(\tilde{K}, v_{0}\right) \approx H$. Thus for a given limit of a group system and a subgroup of that limit we will be studying a covering space of the type of model constructed in $\$$ II. As was noted there such a covering space possesses a 2-stage cover consisting of spaces which are themselves covering spaces of the members of the cover of Theorem II.1. Determining the structure of $H$ will rest in computing the fundamental group of the covering space with respect to the fundamental groups of the members of its cover. For this we will use the version of Van Kampen's theorem given in [1]; we recall its formulation now. For details, see [1].

Suppose that $\Sigma$ is an $n$-stage cover of the complex $K$. For $i=1,2, \ldots, n$ we let $W_{i}$ be the bipartite graph with one class of vertices in 1-1 correspondence with the members of $\lambda_{i-1}$ and the other in 1-1 correspondence with the arc components of $U \lambda_{i}$. Edges are introduced according to nonempty intersection. Maximal trees are then chosen for each of the components of each of the $W_{i}$. Once this has been done the fundamental group of the complex $K$ is given as the limit of a system of groups and homomorphisms whose description is as follows. For each $A \in \Sigma$ there is a group which is the free product of $\pi_{1}(A)$ and $F(D(A))$, a free group on a set of generators $D(A)$. The set $D(A)$ is in $1-1$ correspondence with those edges of the complements of the maximal trees with a vertex corresponding to a member of $\Sigma$ that is contained in $A$. A homomorphism exists between any two groups corresponding to members of $\Sigma$ which are related by containment. For example suppose that $B \subseteq A$. The homomorphism from $\pi_{1}(B) * F(D(B))$ to $\pi_{1}(A) * F(D(A))$ will be the product homomorphism of the homomorphisms induced by the inclusion maps $B \rightarrow A$ and $D(B) \rightarrow D(A)$ followed by the inner automorphism given by the word in $D(A)$ corresponding to the "path" in the $W_{i}$ from $B$ to $A$. While this may prove involved for an $n$-stage cover it will not be too cumbersome for the case of the 2-stage covers considered here. The structure theorem that arises most naturally from the topological setting is then

Theorem III.1. Let $H$ be any subgroup of the limit of the group system $\left(G_{\alpha}, H_{\alpha \beta}, i_{\alpha \beta}: \alpha, \beta \in \Lambda\right)$. Then $H$ is itself the limit of a group system. Each of the factors is the free product of a conjugate of a subgroup of one of the $G_{a}$ and a free group (which may be trivial). Each of the amalgamating groups is the free product of a conjugate of a subgroup of one of the $H_{\alpha \beta}$ and a free group (which may be trivial). 
Proof. Using the construction of Theorem II.1 let $K$ be the complex whose fundamental group is the limit of the system, and let $\left\{K_{\alpha}: \alpha \in \Lambda\right\}$ be the covering of that complex. As was noted there, the $\Sigma$ of this cover will be 2 -stage. Now let $(\tilde{K}, p)$ be the covering space of $K$ which corresponds to the subgroup $H$. By Corollary II.2, $K$ has a 2 -stage cover $\widetilde{\Sigma}$ which consists of the arc components of the inverse images under $p$ of the members of $\Sigma$. The members of $\tilde{\Sigma}$ are themselves covering spaces of the members of $\Sigma$ and as such their fundamental groups correspond to subgroups of the fundamental groups of the members of $\Sigma$. The monomorphism $p_{*}$ will carry them onto conjugates of the subgroups of the corresponding member of the group system below. Since each of the fundamental groups for $\tilde{\lambda}_{2}$ will be trivial (recall $\lambda_{2}$ is the arc $s$ ) the computation of the fundamental group of $\widetilde{K}$ will yield the group described in the statement of the theorem.

We would now like to consider the various forms of this theorem for some of the particular types of group systems that have been considered in an algebraic setting. Since in these cases it is usually required that the various homomorphisms of the group system are monomorphisms we need to check that if the se conditions hold in the representation of $G$ they will hold for $H$ as well. The following proposition is devoted to that end.

Proposition III.2. Let $A, B \in \Sigma$ such that the homomorphism $i_{*}: \pi_{1}(A) \rightarrow$ $\pi_{1}(B)$ induced by inclusion is a monomorphism. Then for any two corresponding members $\widetilde{A}, \widetilde{B}$ of $\widetilde{\Sigma}$ such that $\widetilde{A} \subseteq \widetilde{B}$ the bomomorphism induced by inclusion will also be a monomorphism.

Proof. In this case we have the following commutative diagram:

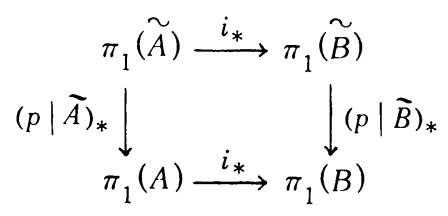

Since $\tilde{A}, \widetilde{B}$ are covering spaces of $A$ and $B$ the homomorphisms $(p \mid \tilde{A})_{*}$, $(p \mid \tilde{B})_{*}$ are monomorphisms; the proposition then follows.

Note that the same type of reasoning applies to $i_{*}: \pi_{1}(A) \rightarrow \pi_{1}(K)$ for $A \in \lambda_{0}$ and $i_{*}: \pi_{1}(\tilde{A}) \rightarrow \pi_{1}(\tilde{K})$ for $\tilde{A}$ over $A$.

In the algebraic setting the considerations have divided themselves along two lines. The first places some restriction on the structure of the original group system such as requiring the amalgamating groups to be trivial (the original theorem of Kurosh) or requiring a single amalgamating subgroup (as considered in [3] and [6]). The second is typified in the original work of H. Neumann [5] 
which makes no restriction on the original group system but results in some final restriction on the subgroups that may be described. In this setting the first kind of restriction can be summarized as the following condition on the original structure. Let $D$ be the diagram of the group system; that is to say a graph with vertices in 1-1 correspondence with the factors and amalgamating groups of the system and with edges in 1-1 correspondence with the homomorphisms of the system. Then $D$ is a tree. This situation is what Karass and Solitar call a tree product in [3]. Since the condition is basically a geometric simplification we should expect that it will yield a simplification in the topological setting. It does, and the form it takes is

Lemma III.3. If the diagram of the group system $\left(G_{a^{\prime}} H_{\alpha \beta}, i_{\alpha \beta}: \alpha, \beta \in \Lambda\right)$ is a tree then there is a complex $K$ with $\pi_{1}(K)$ isomorphic to the limit of the group system. $K$ possesses a 1-stage covering which satisfies the properties of Theorem II.1.

Proof. The construction of $K$ will proceed essentially as in Theorem II.1. However, since the diagram is now a tree the following modification may be made without disturbing the final result. Instead of using the arc $s$, introduce disjoint arcs from each $v_{\alpha \beta}$ to the corresponding $v_{\alpha}$ and $v_{\beta}$. Proceed to attach the necessary discs as before but now the final cover will be 1 -stage.

The importance of a construction which permits the necessary cover to be 1-stage is of course that now, when the computation of the fundamental group of the appropriate covering space has to be made, one only has a 1-stage cover to contend with. In the case of applying this approach to the Kurosh theorem the final result will be a proof almost identical to the one given by Massey in [4]. Before considering what this will yield for free products with a single amalgamated subgroup and the more general tree products, let us examine explicitly what type of group presentation is obtained from a 1-stage cover. It should be noted that Serre [7] has obtained results similar to those contained here by studying such groups explicitly.

Suppose that $K$ has a 1 -stage cover $\Sigma$ with $\lambda_{0}=\left\{K_{\alpha}: \alpha \in \Lambda\right\}$ and $\lambda_{1}=$ $\left\{L_{\alpha \beta}^{\gamma}: \alpha, \beta \in \Lambda\right\}$ where $L_{\alpha \beta}^{\gamma}$ over $\gamma$ is used to denote the various arc components of $K_{\alpha} \cap K_{\beta}$. Suppose that we use $G_{\alpha}$ for $\pi_{1}\left(K_{\alpha}\right)$ and $H_{\alpha \beta}^{\gamma}$ for $\pi_{1}\left(L_{\alpha \beta}^{\gamma}\right)$. Once a maximal tree has been chosen for $W_{1}$ the fundamental group of $K$ will have the group presentation:

$$
\left(\left\{t_{i}\right\},\left\{\text { gen }\left(G_{\alpha}\right)\right\}:\left\{\operatorname{rel}\left(G_{\alpha}\right)\right\},\left\{i_{\alpha \beta}^{\gamma}\left(H_{\alpha \beta}^{\gamma}\right)=i_{\alpha \underline{\beta}}^{\gamma}\left(H_{\alpha \beta}^{\gamma}\right)\right)\right.
$$

where $G_{\alpha}$ has the group presentation $\left(\operatorname{gen}\left(G_{\alpha}\right): \operatorname{rel}\left(G_{\alpha}\right)\right),\left\{t_{i}\right\}$ is the complement of the maximal tree in $W_{1}$, and the homomorphisms $i_{\alpha}^{\gamma}{ }_{\beta}: H_{\alpha \beta} \rightarrow G_{\alpha}$ and 
$i_{\alpha \underline{\beta}}^{\gamma}: H_{\alpha \beta}^{\gamma} \rightarrow G_{\beta}$ are either an inclusion homomorphism or an inclusion homomorphism followed by conjugation by one of the $t_{i}$. So the presentation of $\pi_{1}(K)$ will be of the general form:

$$
\left(\left\{t_{i}\right\},\left\{\text { gen } G_{\alpha}\right\}:\left\{\operatorname{rel} G_{\alpha}\right\},\left\{H_{\alpha} \frac{\gamma}{\beta}=H_{\alpha \bar{\beta}}^{\gamma}\right\},\left\{t_{i} H_{\frac{\alpha}{\alpha} \beta_{i}}^{-1}=H_{\alpha \bar{\beta}}^{\gamma}\right\}\right) .
$$

In [3] Karass and Solitar study the problem of an arbitrary subgroup $H$ of $G=(A * B ; U)$ the free product of $A$ and $B$ with amalgamated subgroup $U . H$ is then determined to be an HNN group of the form

$$
H=\left(t_{1}, t_{2}, \ldots, S \text {; rel } S, t_{1} U_{H}^{\sigma_{1}} t_{1}^{-1}=U_{H}^{\sigma_{1}}, t_{2} U_{H}^{\sigma_{1}}, t_{2}^{-1}=U_{H}^{\sigma_{2}}, \ldots\right)
$$

where $S$ is a tree product with vertices conjugates of $A$ or $B$ intersected with $H$ and associated subgroups conjugates of $U$ intersected with $H$. Comparing (3) with (2) we see that this is precisely what will occur if we take ( $S:$ rel $S$ ) to be the group presentation ( $\left\{\right.$ gen $\left.G_{\alpha}\right\}:\left\{\right.$ rel $\left.G_{\alpha}\right\},\left\{H \frac{\gamma}{\alpha} \beta=H_{\alpha \bar{\beta}}^{\gamma}\right\}$ ). Since we have only been using the fact that the diagram of the system is a tree the appropriate version of the Karass-Solitar the orem will hold for any subgroup of any tree product. Its statement would be

Theorem III.4. Let $H$ be any subgroup of a tree product $G$. Then $H$ is an $H N N$ group whose base is a tree product with vertices which are conjugates of subgroups of the vertices of $G$ and with amalgamating groups which are conjugates of subgroups of the amalgamating groups of $G$. The associated subgroups will also be conjugates of subgroups of the amalgamating groups.

If we compare the statements of Theorem III.1 and Theorem III.4 we notice that, while the subgroup of the limit of an arbitrary group system is itself the limit of a group system, the subgroup of a tree product is not given as a tree product. At first glance this is disappointing but it is only a result of the group presentation that the topology has suggested rather than an algebraic anomaly. There is an alternate group presentation that will give the subgroup as a tree product. The following proposition (restricted to a simple case to avoid complexity) indicates how the adjustment may be made.

Proposition III.5. Let groups $G_{1}, G_{2}$ bave group presentations $\left(\mathbf{a}_{1}: \mathbf{r}_{1}\right)$ and $\left(\mathbf{a}_{2}: \mathbf{r}_{2}\right)$ respectively. Let $\theta_{1}: H_{0} \rightarrow G_{1}$ and $\theta_{2}: H_{0} \rightarrow G_{2}$ be homomorphisms from the group $H_{0}$ which bas a presentation $\left(\mathbf{x}: \mathbf{r}_{0}\right)$. Then the presentation

$$
H=\left(\mathbf{a}_{1}, \mathbf{a}_{2}, t: \mathbf{r}_{1}, \mathbf{r}_{2}, \theta_{1}(\mathbf{x})=t \theta_{2}(\mathbf{x}) t^{-1}\right)
$$

is equivalent to the presentation

$$
H=\left(\mathbf{a}_{1}, \mathbf{a}_{2}, t, y_{\mathbf{x}}: \mathbf{r}_{1}, \mathbf{r}_{2}, \mathbf{r}_{y}, \theta_{1}(\mathbf{x})=t y_{\mathbf{x}} t^{-1}, y_{\mathbf{x}}=\theta_{2}(\mathbf{x})\right) .
$$


As such, $H$ may be considered as a limit with factors $H_{1}, H_{2}$ and another group $\mathrm{H}_{3}$ and with amalgamating group $\mathrm{H}_{0}$.

Proof. By a series of Tietze II operations [2] the original presentation is equivalent to the presentation:

$$
\left(\mathbf{a}_{1}, \mathbf{a}_{2}, t, y_{\mathbf{x}}: \mathbf{r}_{1}, \mathbf{r}_{2}, \theta_{1}(\mathbf{x})=t \theta_{2}(\mathbf{x}) t^{-1}, y_{\mathbf{x}}=\theta_{2}(\mathbf{x})\right)
$$

Applying a series of Tietze I operations to this presentation will yield

$$
\left(\mathbf{a}_{1}, \mathbf{a}_{2}, t, y_{\mathbf{x}}: \mathbf{r}_{1}, \mathbf{r}_{2}, \theta_{1}(\mathbf{x})=t y_{\mathbf{x}} t^{-1}, y_{\mathbf{x}}=\theta_{2}(\mathbf{x})\right) .
$$

Now since $\theta_{2}$ is a homomorphism, for any relation, $r$, in $\mathbf{r}_{0}, \theta_{2}(r)$ will be in the consequence of $\mathbf{r}_{2}$ and may be added along with its conjugate by $t$ (both expressed in terms of the $y_{\mathbf{x}}$ ) to the above relation set via a series of Tietze I operations. Let $\mathbf{r}_{y}$ denote the set of relations so added to obtain

$$
H=\left(\mathbf{a}_{1}, \mathbf{a}_{2}, t, y_{\mathbf{x}}: \mathbf{r}_{1}, \mathbf{r}_{2}, \mathbf{r}_{y}, \theta_{1}(\mathbf{x})=t y_{\mathbf{x}} t^{-1}, y_{\mathbf{x}}=\theta_{2}(\mathbf{x})\right) .
$$

Now let $H_{3}$ be the group with the presentation $\left(y_{\mathbf{x}}, t: \mathbf{r}_{y}\right)$, then the functions $x \rightarrow y_{x}, x \rightarrow f y_{x} f^{-1}$ induce homomorphisms $\theta_{3}: H_{0} \rightarrow H_{3}$ and $\theta_{4}: H_{0} \rightarrow H_{3}$ by the way we constructed $\mathbf{r}_{y}$. This gives $H$ then as the limit of the group system described.

One may ask as well whether in the case of a free product with a single amalgamated subgroup the subgroup can also be described as a free product with a single amalgamated subgroup rather than an HN group or tree product. This question is answered in the affirmative by Ordman in [6]. This treatment of the problem yields a description of the subgroup as the free product with single amalgamated subgroup, each of whose factors is generated by conjugates of subgroups of the corresponding factor of the original system, and whose amalgamating group is generated by conjugates of subgroups of the original amalgamating group. In the setting of this paper such a description would arise by choosing a different covering for the computation of the fundamental group of $\tilde{K}$. In the case of a single amalgamated subgroup, the construction of the topological model $K$ would have a cover $\left\{K_{a}: a \in \Lambda\right\}$ with each $K_{a} \cap K_{\beta}=L$, with $\pi_{1}(L)$ being the amalgamating subgroup. For the $(\tilde{K}, p)$ corresponding to the subgroup one could choose a covering as follows. Let $T$ be a maximal tree in $\underset{\sim}{\mathcal{K}} \underset{\sim}{\sim}$ and let $\widetilde{K}_{\alpha}=T$ $\cup\left\{\right.$ arc components of $\left.p^{-1}\left(K_{\alpha}\right)\right\}$. Then this covering has $\widetilde{K}_{a} \cap \widetilde{K}_{\beta}=T \cup\{$ arc components of $\left.p^{-1}(L)\right\}$ and $W_{1}$ being the complete bipartite graph $K_{1, \Lambda}$. This will give $\pi_{1}(K)$ as the type of free product with amalgamated subgroup that Ordman obtains. The interest here is that the differences in the description arise topologically as opposed to algebraically.

We now turn our attention to the second type of algebraic situation as 
typified by the original work of $\mathrm{H}$. Neumann [5]. Recall that now no restrictions will be made upon the type of the original group system. The description of the subgroup $H$ which was obtained was that of the limit of a group system. The factors were either conjugates of subgroups of the original system or another type which had a conjugate of a subgroup of an original factor as a normal subgroup with factor group $Z$ by this normal subgroup. The amalgamating groups were conjugates of subgroups of the original amalgamating groups. There was also the condition that $H$ not be contained in the smallest subgroup of the original group that was itself a limit of a group system with the same amalgamating subgroups. In Proposition III.5 we have already seen the origin of the other type of factor in the description of the subgroup, so we will focus our attention on the condition placed on the subgroup $H$. Since Theorem IV.1 already describes $H$ as the limit of a group system in terms of factors and amalgamating subgroups that are free products of conjugates of subgroups of the original system and certain free groups, let us now try for a description of $H$ that permits only conjugates of subgroups of the amalgamating groups as amalgamating groups for $H$.

Recall that now with no restriction on the original group system we will be dealing with a 2 -stage cover in the computation of $\pi_{1}(\widetilde{K})$. The algebraic conditions that have been set place the topological condition on the 2-stage cover that the components of $W_{2}$ be trees while the components of $W_{1}$ may be arbitrary. For $\tilde{\Sigma}, \tilde{\lambda}_{2}$ consists of the arc components of $p^{-1}(s)$, thus $W_{2}$ consists of one class of vertices in 1-1 correspondence with the arc components of $p^{-1}\left(L_{\alpha \beta} \cup\right.$ arc from $v_{\alpha \beta}$ to $\left.v_{0}\right)$ and the other in 1-1 correspondence with $p^{-1}(s)$. For the discussion that follows assume that the base point of $K$ is $v_{0}$. Suppose that $C$ were an arc component of $W_{2}$ which contains a nontrivial path $\alpha$. Since $W_{2}$ is a bipartite graph, a may be factored into a collection of paths $e_{i}, e_{i}^{\prime}$ where $e_{i}$ is an edge from a component of $p^{-1}(s)$ to a component of $p^{-1}\left(L_{\alpha \beta} \cup\right.$ arc from $v_{\alpha \beta}$ to $\left.v_{0}\right)$ and $e_{i}^{\prime}$ is an edge from that same component back to another component of $p^{-1}(s)$. Accordingly we may choose an arc $\gamma_{i}$ in each such component such that $\gamma_{i}$ corresponds to $e_{i}$, $e_{i}^{\prime}$. Since $\gamma_{i}$ is a path in $\widetilde{K}$ while $p\left(\gamma_{i}\right)$ is a loop in $\left(L_{\alpha \beta} \cup\right.$ arc from $v_{\alpha \beta}$ to $\left.v_{0}\right), \gamma_{i}$ corresponds to an element of one of the amalgamating subgroups which is not a member of the group $H$. Therefore a corresponds to a member of $H$ (a loop in $\widetilde{K}$ ) which factors into a product of elements from the amalgamating groups none of which belong to $H$.

In summary then we have proved (taking into account components of $W_{2}$ which do not contain the basepoint of $\widetilde{K}$ )

Proposition III.6. If $H$ contains the corresponding conjugates of the individual factors of any conjugate in $H$ of a product of elements from the 
amalgamating groups of $G$, then the amalgamating groups of $H$ consist only of conjugates of subgroups of the amalgamating groups of $G$.

In the case of the arbitrary group system, then, the topological approach matches the algebraic treatment with regard to the factors of the subgroup but differs in the description of the amalgamating groups. Either all subgroups can be described using free products of conjugates of subgroups of the original amalgamating groups and a free group, or some subgroups may be described using only conjugates of subgroups of the original amalgamating groups. In the latter case the topological condition and the algebraic condition differ. We would conjecture that a match cannot be made here by merely choosing a different covering for $\widetilde{K}$ as was done before. It would probably be necessary to give an alternate construction of $K$ which would minimize the use of the arc $s$ to those situations where it was absolutely necessary to permit the construction of the desired complex.

\section{REFERENCES}

1. J. C. Chipman, Van Kampen's theorem for n-stage covers, Trans. Amer. Math. Soc. (to appear).

2. R. H. Crowell and R. H. Fox, Introduction to knot theory, Ginn, Boston, Mass., 1963. MR $26 \# 4348$.

3. A. Karrass and D. Solitar, The subgroups of a free product of two groups with an amalgamated subgroup, Trans. Amer. Math. Soc. 150 (1970), 227-255. MR 41 \#5499.

4. W. S. Massey, Algebraic topology: An introduction, Harcourt, Brace \& World, New York, 1967. MR $35 \# 2271$.

5. H. Neumann, Generalized free products with amalgamated subgroups. II, Amer. J. Math. 71 (1949), 491-540. MR 11, 8.

6. E. T. Ordman, On subgroups of amalgamated free products, Proc. Cambridge Philos. Soc. 69 (1971), 13-23. MR 43 \#2102.

7. J. Serre, Groupes discrets, Notes written at Collège de France, 1968/69.

DEPARTMENT OF MATHEMATICS, OAKLAND UNIVERSITY, ROCHESTER, MICHIGAN 48063 